\title{
Conformal equivalence of visual metrics in pseudoconvex domains
}

\author{
Luca Capogna ${ }^{1} \cdot$ Enrico Le Donne ${ }^{2,3}$ (i)
}

Received: 19 December 2018 / Revised: 20 January 2020 / Published online: 3 February 2020

(c) The Author(s) 2020

\begin{abstract}
We refine estimates introduced by Balogh and Bonk, to show that the boundary extensions of isometries between bounded, smooth strongly pseudoconvex domains in $\mathbb{C}^{n}$ are conformal with respect to the sub-Riemannian metric induced by the Levi form. As a corollary we obtain an alternative proof of a result of Fefferman on smooth extensions of biholomorphic mappings between bounded smooth pseudoconvex domains. The proofs are inspired by Mostow's proof of his rigidity theorem and are based on the asymptotic hyperbolic character of the Kobayashi or Bergman metrics and on the Bonk-Schramm hyperbolic fillings.
\end{abstract}

Mathematics Subject Classification $32 \mathrm{~T} 15 \cdot 32 \mathrm{Q} 45 \cdot 32 \mathrm{H} 40 \cdot 53 \mathrm{C} 23 \cdot 53 \mathrm{C} 17$

Communicated by Ngaiming Mok.

Luca Capogna was partially funded by NSF award DMS 1503683 and Simons collaboration grant for mathematicians 585688.

Enrico Le Donne was partially supported by the Academy of Finland (Grant 288501 'Geometry of subRiemannian groups' and by grant 322898 'Sub-Riemannian Geometry via Metric-geometry and Lie-group Theory') and by the European Research Council (ERC Starting Grant 713998 GeoMeG 'Geometry of Metric Groups').

$凶$ Enrico Le Donne

enrico.ledonne@unipi.it

Luca Capogna

lcapogna@wpi.edu

1 Department of Mathematical Sciences, Worcester Polytechnic Institute, Worcester, MA 01609, USA

2 Dipartimento di Matematica, Università di Pisa, Largo B. Pontecorvo 5, 56127 Pisa, Italy

3 Department of Mathematics and Statistics, University of Jyväskylä, P.O. Box (MaD), 40014 Jyväskylä, Finland 


\section{Introduction}

Let $D \subset \mathbb{C}^{n}(n \geq 2)$ be a bounded, strongly pseudo-convex domain with $C^{\infty}$-smooth boundary. Denote by $d_{K}$ the distance function corresponding to a Finsler structure $K$ satisfying suitable estimates, see (2.8). For example, one may consider the Bergman metric or the Kobayashi metric or the inner Carathéodory metric.

In $[2,3]$, Balogh and Bonk have proved that the metric space $\left(D, d_{K}\right)$ is hyperbolic in the sense of Gromov and its visual boundary coincides with the topological boundary $\partial D$. They also show that the Carnot-Carathéodory metric $d_{C C}$ corresponding to the Levi form on $\partial D$, determines the canonical class of snowflake equivalent visual metrics on $\partial D$. As a consequence, results from the theory of Gromov hyperbolic spaces can be immediately applied in this setting. Among these we recall that every quasi-isometry between such spaces extends to a quasi-conformal map between the visual boundaries, endowed with their families of visual metrics, see for instance $[6,17]$ and references therein.

Our main contribution is to show that extensions of isometries are actually diffeomorphisms that are conformal with respect to the Carnot-Carathéodory metric. We only need to show that the extension is 1-quasi-conformal, as the smoothness then follows from the recent results in [12].

As in [3], our strategy involves the Bonk-Schramm hyperbolic filling metric $g$ defined in (1.2). This metric provides a stepping stone to connect the CarnotCarathéodory distance, defined on the boundary by the Levi form (see Sect. 2.2), with the invariant metric defined in the domain.

Theorem 1.1 Let $D_{1}, D_{2} \subset \mathbb{C}^{n}$ be bounded strongly pseudoconvex $C^{\infty}$-smooth domains and denote by $d_{K}$ the distance function corresponding to a Finsler structure $K$ satisfying (2.8), and by $d_{C C}$ the Carnot-Carathéodory distance on the boundaries induced by the Levi form. If $f:\left(D_{1}, d_{K}\right) \rightarrow\left(D_{2}, d_{K}\right)$ is an isometry then the induced boundary map $F:\left(\partial D_{1}, d_{C C}\right) \rightarrow\left(\partial D_{2}, d_{C C}\right)$ is a diffeomorphism, conformal with respect to the metric $d_{C C}$.

We emphasize that the result holds when $d_{K}$ is the Bergman, the Kobayashi, or the inner Carathéodory metrics. Indeed, these distances satisfy (2.8) in view of the work in $[2,3,21]$.

As we noted above, the proof of Theorem 1.1 is based on the study of the relation between the visual distances associated to $d_{K}$ and the visual distance of an ad-hoc hyperbolic filing metric, built through the Carnot-Carathéodory distance: For $x \in D$ denote by $h(x):=\sqrt{d_{E}(x, \partial D)}$ and by $\pi(x) \in \partial D$ a closest point in $\partial D$ with respect to the Euclidean distance $d_{E}(\cdot, \cdot)$, noting it is uniquely defined in a neighborhood of วD. Set

$$
g(x, y):=2 \log \left(\frac{d_{\mathrm{CC}}(\pi(x), \pi(y))+\max (h(x), h(y))}{\sqrt{h(x) h(y)}}\right) .
$$

This is an hyperbolic filling metric built from the metric space $\left(\partial D, d_{\mathrm{CC}}\right)$ (see Bonk and Schramm [10]). Balogh and Bonk [3, Corollary 1.3], showed that $g$ is a metric in 
a neighborhood of $\partial D$ and that $g$ and the invariant distance function $d_{K}$ are $(1, C)$ quasi-isometric. As a consequence, they give rise to quasi-conformally equivalent visual metrics.

The main technical point of our work is to refine this result in a quantitative fashion. We show that a particular visual quasi-distance $\rho_{o}^{K}$ associated to the invariant metric $d_{K}$ is in fact pointwise and asymptotically $(1+\epsilon)$-quasi-conformally equivalent to the Carnot-Carathéodory $d_{C C}$ metric. By pointwise and asymptotically we mean that for every point $x \in \partial D$ in the boundary, and for every $\epsilon>0$, one can choose a base point $o$ for the definition of the visual distances so that the identity map has distortion less than $1+\epsilon$ at $x$. Following ideas in CAT $(-1)$ spaces, given a pointed metric space $(X, d, o)$ we consider the visual function

$$
\rho_{o}^{d}(x, y)=\exp \left(-\langle x, y\rangle_{o}\right)
$$

where $\langle x, y\rangle_{o}$ denotes the Gromov product in $(X, d)$, see Sect. 2. Usually, $\rho_{o}^{d}$ is called Bourdon distance since for CAT $(-1)$ spaces it satisfies the triangle inequality. In our setting, $\rho_{o}^{d}$ may not be a distance.

Moreover, Bourdon showed in [9] that on a CAT $(-1)$ space $X$ the visual boundaries $\left(\partial_{\infty} X, \rho_{o}^{d}\right)$ corresponding to different base points $o, o^{\prime} \in X$ are conformally equivalent, thus implying immediately that any isometry of $X$ extends to a conformal maps of its visual boundaries. Since pseudoconvex domains may not have negative curvature (see [19]) and may not be simply connected, they are not CAT $(-1)$ spaces and so one cannot apply Bourdon's result.

Theorem 1.1 is achieved in two steps: First one shows that the Carnot-Carathéodory distance is conformally equivalent ${ }^{1}$ to the function $\rho_{o}^{g}$ associated to the hyperbolic filling metric $g$.

Proposition 1.4 For any $o \in D$, the functions $d_{C C}$ and $\rho_{o}^{g}$ are conformally equivalent.

In other words, the identity map $\left(\partial D, d_{C C}\right) \rightarrow\left(\partial D, \rho_{o}^{g}\right)$ has distortion that is identically equal to one. See (2.1) for the definition of distortion.

Next, we show that at every boundary point, and for any $\epsilon>0$, one can find a base point $o \in D$ such that the corresponding visual functions $\rho_{o}^{K}$ and $\rho_{o}^{g}$ are $(1+\epsilon)$ biLipschitz equivalent in a neighborhood of that point. In the following we denote Euclidean balls in $\mathbb{C}^{n}$ with the notation $B(x, r)$.

Proposition 1.5 For any $\bar{p} \in \partial D$ and $\bar{\epsilon}>0$ there exists $r>0$ such that for all $\omega \in \partial D \cap B(\bar{p}, r) \backslash\{\bar{p}\}$ there exists $r^{\prime}>0$ such that for all $o \in D \cap B\left(\omega, r^{\prime}\right)$ the two functions $\rho_{o}^{g}$ and $\rho_{o}^{K}$ are $(1+\bar{\epsilon})$-biLipschitz on $\partial D \cap B\left(\bar{p}, r^{\prime}\right)$.

The proof of Proposition 1.5 and Theorem 1.1 are in Sect. 5. Theorem 1.1 follows rather directly from Propositions 1.4 and 1.5 and from the following diagram

1 The result holds for any hyperbolic filling as in the work of Bonk and Schramm. See Sect. 3.1 


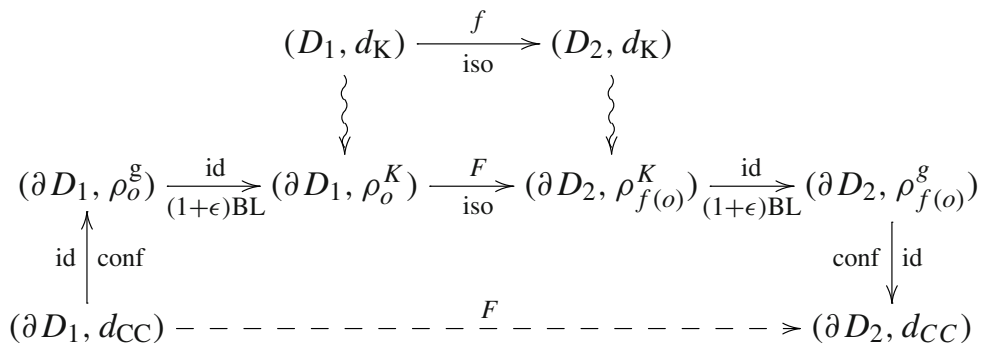

At the center of this chain of compositions there is an isometry, the rest of the links are either $(1+\epsilon)$-biLipschitz maps or conformal maps, so that the total distortion is at most $\epsilon$ away from being equal to 1 everywhere.

From the conformal equivalence theorem above and the results in [12], one can immediately infer a result about boundary extensions for biholomorphisms between strictly pseudoconvex domains in $\mathbb{C}^{n}$, originally established by Fefferman [15].

Corollary 1.6 Let $D_{1}, D_{2} \in \mathbb{C}^{n}(n \geq 2)$ be bounded strongly pseudo-convex domains with $C^{\infty}$-smooth boundaries. If $f: D_{1} \rightarrow D_{2}$ is a biholomorphism then it extends to a smooth map $F: \partial D_{1} \rightarrow \partial D_{2}$ that is conformal with respect to the corresponding subRiemannian contact structure. In particular, at every boundary point, its differential is a similarity between the maximally complex tangent planes.

Since the publication of [15] there have been several significative extensions and simplifications of the result. A small sample of this extensive line of inquiry can be found in the references $[1,4,5,8,13,20,22]$.

The contribution of the present paper does not lie so much in an innovation on a technical level, but rather in two new insights: namely, that one can deduce Fefferman's result from the conformality of the boundary extension and that one can prove this with relative ease from a combination of the general theory of Gromov hyperbolic spaces in combination with careful estimates in Theorem 4.1.

We conclude by observing that our work can be seen as an instance of a dictionary, introduced by Bonk, Heinonen, and Koskela in [7], translating back and forth problems in domains in Euclidean spaces by means of ad hoc hyperbolic or quasi-hyperbolic metrics, that endow such domains with an hyperbolic structure in the sense of Gromov. For more results along this line, see also the recent, interesting work of Zimmer in [24].

\section{Preliminaries}

In this section we recall some basic definitions and results. We start by discussing distortion and conformal maps on subRiemannian manifolds. Then we discuss pseudoconvex domains and their metrics. Finally we review hyperbolicity in the sense of Gromov. 


\subsection{Distorsion in subRiemannian geometry}

By a previous work of the authors together with Ottazzi, we know that several definitions of conformal maps are equivalent in the setting of contact subRiemannian manifolds. We now recall the two definitions that we shall need in this paper.

For a homeomorphism $F: X \rightarrow Y$ between general metric spaces, we consider the following quantities

$$
\mathrm{L}_{F}(x):=\limsup _{x^{\prime} \rightarrow x} \frac{d\left(F(x), F\left(x^{\prime}\right)\right)}{d\left(x, x^{\prime}\right)} \quad \text { and } \quad \ell_{F}(x):=\liminf _{x^{\prime} \rightarrow x} \frac{d\left(F(x), F\left(x^{\prime}\right)\right)}{d\left(x, x^{\prime}\right)}
$$

The quantity $\mathrm{L}_{F}(x)$ is sometimes denoted by $\operatorname{Lip}_{F}(x)$ and is called the pointwise Lipschitz constant. Within this paper, we define the distortion of $f$ at a point $x \in X$ as

$$
H^{*}\left(x, F, d_{X}, d_{Y}\right):=\frac{\mathrm{L}_{F}(x)}{\ell_{F}(x)} .
$$

The homeomorphism $f$ is said to be quasi-conformal if there exists $K$ such that for all $x \in X$ one has

$$
\limsup _{r \rightarrow 0} \frac{\sup \left\{d_{Y}(F(p), F(q)): d_{X}(p, q) \leq r\right\}}{\inf \left\{d_{Y}(F(p), F(q)): d_{X}(p, q) \geq r\right\}} \leq K
$$

It is well-known that in the literature there are several other equivalent definitions of quasi-conformality in 'geometrically nice' spaces, see [23]. However, the equivalence is not quantitative, in the sense that each definition has an associated constant (like the $K$ above) and the value of of these constants can be different from definition to definition. Thus we need to clarify what is a conformal map. To do this we invoke some recent results due to Citti, Ottazzi and the authors of the present paper.

Lemma 2.2 [12, Theorem 1.3 and Theorem 1.19] Let $F: X \rightarrow Y$ be a quasiconformal homeomorphism between two equiregular subRiemannian manifolds.

(i) The requirement $H^{*}\left(\cdot, F, d_{X}, d_{Y}\right) \equiv 1$ is equivalent to other notions of 1-quasiconformality.

(ii) If $X$ and $Y$ are contact manifolds, then 1-quasi-conformality of $F$ is equivalent to $F$ being conformal (i.e., smooth and with horizontal differential that is a homothety).

One of the advantages to work with (2.1) is that it immediately yields a chain rule:

$$
H^{*}\left(x, F_{1} \circ F_{2}\right) \leq H^{*}\left(x, F_{2}\right) H^{*}\left(F_{2}(x), F_{1}\right) .
$$

The last equation follows from the fact that $\lim \sup a_{n} b_{n} \leq \lim \sup a_{n} \lim \sup b_{n}$ whenever $a_{n}, b_{n} \geq 0$. Moreover, we trivially have that if $F$ is an $L$-biLipschitz homeomorphism, then

$$
H^{*}(x, F) \leq L^{2} .
$$




\subsection{Pseudoconvex domains and hermitian metrics}

We recall some of the basic definitions about pseudoconvex domains and hermitian metrics, as well as some key results proved by Balogh and Bonk in [3].

Let $D \subset \mathbb{C}^{n}, n \geq 2$ be a smooth, bounded open set. Let $\varphi: \mathbb{C}^{n} \rightarrow \mathbb{R}$ denote the signed distance function from $\partial D$, negative in $D$ and positive in its complement. Set $N_{\delta}=\left\{x \in D \mid d_{E}(x, \partial D)<\delta\right\}$.

Lemma 2.5 (Tubular Neighborhood Theorem) Let $D \subset \mathbb{C}^{n}, n \geq 2$ be a bounded domain with smooth boundary. There exists $\delta_{0}>0$ such that the projection $\pi$ : $N_{\delta_{o}} \rightarrow \partial D$ is a smooth, well defined map and the distance function $d_{E}(\cdot, \partial D)$ is smooth on $N_{\delta_{0}}$.

We will denote by $n(x)$ the outer unit normal at $x \in \partial D$, so that the fiber $\pi^{-1}(x) \cap$ $N_{\delta_{0}}=\left\{x+\operatorname{sn}(x) \mid s \in\left(0, \delta_{0}\right)\right\}$.

For $p \in \partial D$, one can define the tangent space $T_{p} \partial D=\left\{Z \in \mathbb{C}^{n} \mid \operatorname{Re}\langle\bar{\partial} \varphi(p), Z\rangle=\right.$ $0\}$ and its maximal complex subspace $H_{p} \partial D=\left\{Z \in \mathbb{C}^{n} \mid\langle\bar{\partial} \varphi(p), Z\rangle=0\right\}$, where $\left\langle Z, Z^{\prime}\right\rangle=\sum_{i=1}^{n} Z_{i} \bar{Z}_{i}^{\prime}$ is the hermitian product. By definition, the domain $D$ is strictly pseudoconvex if for every $p \in \partial D$, the Levi form

$$
L_{\varphi}(p, Z):=\sum_{\alpha, \beta=1}^{n} \partial_{z_{\alpha} \bar{z}_{\beta}}^{2} \varphi(p) Z_{\alpha} \bar{Z}_{\beta}
$$

is positive definite on $H_{p} \partial D$.

For each $p \in \partial D$ one has a splitting $\mathbb{C}^{n}=H_{p} \partial D \oplus N_{p} \partial D$, where $N_{p} \partial D$ is the complex one-dimensional subspace orthogonal to $H_{p} \partial D$. This splitting at $p$ induces a decomposition $Z=Z_{H}+Z_{N}$ for all $Z \in \mathbb{C}^{n}$.

Metrics that are invariant under the action of biholomorphisms play a key role in several complex variables. Important examples are the Bergman metric, the Kobayashi metric, and the inner Carathéodory metric (see [19]). In all cases, for $x \in D$ the length of a complex vector $Z \in T_{x} D=\mathbb{C}^{n}$ is given by a Finsler structure $K(x, Z)$. We will rely on the following result, which can be found (along with more references) in [2] and also [11], [14], [16], and [3, Proposition 1.2].

Proposition 2.7 (Balogh-Bonk) Let $D \subset \mathbb{C}^{n}, n \geq 2$ be a bounded, strictly pseudoconvex domain with smooth boundary and let $K(x, Z)$ be the Finsler structure associated to the Bergman metric or the Kobayashi metric or the inner Carathéodory metric. For every $\bar{\epsilon}>0$ there exists $\delta_{0}, C>0$ such that for all $x \in D$ with $d_{E}(x, \partial D) \leq \delta_{0}$ and $Z \in \mathbb{C}^{n}$ one has

$$
\begin{aligned}
& \left(1-C \sqrt{d_{E}(x, \partial D)}\right)\left(\frac{\left|Z_{N}\right|^{2}}{4 d_{E}^{2}(x, \partial D)}+(1-\bar{\epsilon}) \frac{L_{\varphi}\left(\pi(x), Z_{H}\right)}{d_{E}(x, \partial D)}\right)^{\frac{1}{2}} \leq K(x, Z) \\
& \leq\left(1+C \sqrt{d_{E}(x, \partial D)}\right)\left(\frac{\left|Z_{N}\right|^{2}}{4 d_{E}^{2}(x, \partial D)}+(1+\bar{\epsilon}) \frac{L_{\varphi}\left(\pi(x), Z_{H}\right)}{d_{E}(x, \partial D)}\right)^{\frac{1}{2}},
\end{aligned}
$$

where $Z=Z_{H}+Z_{N}$ is the splitting at $\pi(x)$. 
The subbundle $H \partial D$ is a contact distribution on $\partial D$ and the triplet ( $\partial D, H \partial D, L_{\varphi}$ ) yields a contact subRiemannian manifold. In this structure, the horizontal curves are those arcs in $\partial D$ that are tangent to the contact distribution, and the CarnotCarathéodory distance $d_{C C}(p, q)$ between $p, q \in \partial D$ is defined as the minimum time it takes to reach one point from the other traveling along horizontal curves at unit speed with respect to the Levi form, see [18].

As in [3], we will need to use a family of Riemannian metrics on $\partial D$ that approximate the sub-Riemannian metric associated to the Levi form, and that in fact have corresponding distance functions that converge in the sense of Gromov-Hausdorff to the Carnot-Carathéodory distance. For every $k>0$ we define a Riemannian metric $g_{k}$ on $T \partial D$ as

$$
g_{k}^{2}(p, Z):=L_{\varphi}\left(p, Z_{H}\right)+k^{2}\left|Z_{N}\right|^{2},
$$

for every $p \in \partial D$ and every $Z=Z_{H}+Z_{N} \in T_{p} \partial D$. Here we just recall a basic comparison result (see for instance [3, Lemma 3.2]) relating the distance function $d_{k}$ associated to $g_{k}$ to the Carnot-Carathéodory distance $d_{C C}$.

Lemma 2.10 There exists a constant $C>0$ such that for all $k>0$, and for all points $p, q \in \partial D$, with $d_{C C}(p, q) \geq k^{-1}$ one has

$$
C^{-1} d_{k}(p, q) \leq d_{C C}(p, q) \leq C d_{k}(p, q)
$$

\subsection{Gromov hyperbolicity}

Let $x, y, o$ be three points in a metric space $(X, d)$. Then the Gromov product of $x$ and $y$ at $o$, denoted $\langle x, y\rangle_{o}$, is defined by

$$
\langle x, y\rangle_{o}=\frac{1}{2}(d(x, o)+d(y, o)-d(x, y))
$$

Then $X$ is called Gromov hyperbolic if there exists $\delta \geq 0$ such that

$$
\langle x, y\rangle_{o} \geq \min \left\{\langle x, z\rangle_{o},\langle z, y\rangle_{o}\right\}-\delta, \quad \text { for all } x, y, z, o \in X
$$

For a Gromov hyperbolic space $X$ one can define a boundary set $\partial_{\infty} X$ as follows, see [6, p.431-2]. Fix a base point $o \in X$. A sequence $\left(x_{i}\right)$ in $X$ is said to converge at infinity if $\lim _{i, j \rightarrow \infty}\left\langle x_{i}, x_{j}\right\rangle_{o}=\infty$. Two sequences $\left(x_{i}\right)$ and $\left(y_{i}\right)$ converging at infinity are called equivalent if $\lim \left\langle x_{i}, y_{i}\right\rangle_{o}=\infty$. These notions do not depend on the choice of the base point $o$. The set $\partial_{\infty} X$ is now defined as the set of equivalence classes of sequences converging at infinity.

For $p, q \in \partial_{\infty} X$ and $o \in X$ we define

$$
\langle p, q\rangle_{o}=\sup \liminf _{i \rightarrow \infty}\left\langle x_{i}, y_{i}\right\rangle_{o}
$$


where the supremum is taken over all sequences $\left(x_{i}\right)$ and $\left(y_{i}\right)$ representing the boundary points $p$ and $q$, respectively. Actually, there exists such sequences $\left(x_{i}\right)$ and $\left(y_{i}\right)$ for which $\langle p, q\rangle_{o}=\lim _{i \rightarrow \infty}\left\langle x_{i}, y_{i}\right\rangle_{o}$, see [6, Remark 3.17].

Balogh and Bonk have proved that if $D \subset \mathbb{C}^{n}, n \geq 2$ is a bounded, strictly pseudoconvex domain with smooth boundary, and $K(x, Z)$ is a norm satisfying (2.8), then the corresponding metric space $\left(D, d_{K}\right)$ is Gromov hyperbolic and its visual boundary coincides with the topological boundary. See [3, Theorem 1.4].

\section{Conformal equivalence of boundary metrics}

\subsection{Boundary distances of hyperbolic fillings}

An important contribution of Bonk and Schramm [10], is that the functor $X \rightarrow \partial_{\infty} X$ has an inverse functor, in the form of hyperbolic filling spaces $C o n(Z)$. To be more precise, one defines $\operatorname{Con}(Z)=Z \times(0, D)$, endowed with the metric given by

$$
g((x, u),(y, v))=2 \log \left(\frac{d_{1}(x, y)+\max (u, v)}{\sqrt{u v}}\right) .
$$

The space $(\operatorname{Con}(Z), g)$ is Gromov hyperbolic, and its visual boundary is $Z$, with the canonical class of snowflake equivalent metrics given by $d_{1}$. In this section we prove that a particular visual metric, i.e. the visual metric generated by $g$ through the formula (1.3), is actually conformal to $d_{1}$. Choose a generic base point $o=(z, s)$, with $z \in Z$ and $s \in(0, D)$. For any two points $x, y \in Z$ so that $d_{1}(x, y)<s$. consider $u, v \in\left(0, d_{1}(x, y)\right)$. Following (1.3), we let $d_{2}(x, y)$ be defined as follows

$$
d_{2}(x, y)=\lim _{u, v \rightarrow 0} e^{-\langle(x, u),(y, v)\rangle_{o}} .
$$

Notice that in general, functions defined as in (1.3), associated to the hyperbolic fillings are a quasi-distance. By quasi-distance we intend that the triangle inequality is satisfied modulo a multiplicative constant.

Proposition 3.2 Let $d_{1}$ a distance on a bounded space Z. If $d_{2}$ is defined as in (1.3), associated to the hyperbolic filling for $d_{1}$, then $d_{1}$ and $d_{2}$ are conformally equivalent.

Proof In order to show that $d_{1}, d_{2}$ are conformally equivalent it suffices to prove that the $\operatorname{limit}_{\lim _{y \rightarrow x}} d_{1}(x, y) / d_{2}(x, y)$ exists for every $x \in Z$. Fix any $z \in Z$ and $s \in(0, D)$. Let $o=(z, s)$. Take two points $x, y \in Z$ so that $d_{1}(x, y)<s$. Take $u, v \in\left(0, d_{1}(x, y)\right)$.

The rest of the proof follows from

$$
\begin{aligned}
\langle(x, u),(y, v)\rangle_{o} & =\frac{1}{2}\left(d_{2}((x, u), o)+d_{2}((y, v), o)-d_{2}((x, u),(y, v))\right) \\
& =\log \left(\frac{d_{1}(x, z)+\max (u, s)}{\sqrt{u s}}\right)+\log \left(\frac{d_{1}(y, z)+\max (v, s)}{\sqrt{v s}}\right)
\end{aligned}
$$




$$
\begin{aligned}
& -\log \left(\frac{d_{1}(x, y)+\max (u, v)}{\sqrt{u v}}\right) \\
= & \log \frac{\left(d_{1}(x, z)+s\right)\left(d_{1}(y, z)+s\right)}{s\left(d_{1}(x, y)+\max (u, v)\right)} \\
= & \log \left(\frac{\left(d_{1}(x, z)+s\right)\left(d_{1}(y, z)+s\right)}{s\left(d_{1}(x, y)+\max (u, v)\right)} \frac{d_{1}(x, y)}{d_{1}(x, y)}\right) \\
= & -\log \left(d_{1}(x, y)\right)+\log \left(\frac{d_{1}(x, y)\left(d_{1}(x, z)+s\right)\left(d_{1}(y, z)+s\right)}{s\left(d_{1}(x, y)+\max (u, v)\right)}\right)
\end{aligned}
$$

We calculate $\lim _{y \rightarrow x} d_{1}(x, y) / d_{2}(x, y)$. Consider the quotient

$$
\begin{aligned}
d_{2}(x, y) / d_{1}(x, y) & =\lim _{u, v \rightarrow 0} \frac{e^{-\langle(x, u),(y, v)\rangle_{o}}}{d_{1}(x, y)} \\
& =\lim _{u, v \rightarrow 0} \frac{e^{\log \left(d_{1}(x, y)\right)} e^{-\log \left(\frac{d_{1}(x, y)\left(d_{1}(x, z)+s\right)\left(d_{1}(y, z)+s\right)}{s\left(d_{1}(x, y)+\max (u, v)\right)}\right)}}{d_{1}(x, y)} \\
& =\lim _{u, v \rightarrow 0}\left(\frac{d_{1}(x, y)\left(d_{1}(x, z)+s\right)\left(d_{1}(y, z)+s\right)}{s\left(d_{1}(x, y)+\max (u, v)\right)}\right)^{-1} \\
& =\frac{s d_{1}(x, y)}{d_{1}(x, y)\left(d_{1}(x, z)+s\right)\left(d_{1}(y, z)+s\right)} \\
& =\frac{s}{\left(d_{1}(x, z)+s\right)\left(d_{1}(y, z)+s\right)}
\end{aligned}
$$

The latter implies that

$$
\lim _{y \rightarrow x} \frac{d_{2}(x, y)}{d_{1}(x, y)}=\frac{s}{\left(d_{1}(x, z)+s\right)^{2}},
$$

which gives the conclusion.

As a corollary of the previous result, one immediately has a proof for Proposition 1.4. In fact, by setting $d_{1}=d_{\mathrm{CC}}$ and applying Proposition 3.2 it follows that the identity map $\left(\partial D, d_{C C}\right) \rightarrow\left(\partial D, \rho_{o}^{g}\right)$ is 1-quasi-conformal.

For possible future reference, we conclude this section by providing an explicit formula for the "distance" function $\rho_{o}^{g}$, where $g$ is as defined in (1.2) and $\rho_{o}^{g}$ is its visual metric as defined in (1.3). We represent $p$ and $q$ by two sequences $x_{i}$ and $y_{i} \in D$, respectively. Notice that since $x_{i} \rightarrow p$ in $\mathbb{C}^{n}$ then $\pi\left(x_{i}\right) \rightarrow p$ and $h\left(x_{i}\right) \rightarrow 0$. In particular, we also have that $\max \left(h\left(x_{i}\right), h(o)\right)=h(o)$. Similar considerations apply to $y_{i}$ and $q$. Elementary computations yield the following

$$
\begin{aligned}
\rho_{o}^{g}(p, q) & =\exp \left(-\langle p, q\rangle_{o}\right) \\
& =\lim _{i \rightarrow \infty} \exp \left(-\left\langle x_{i}, y_{i}\right\rangle_{o}\right) \\
& =\lim _{i \rightarrow \infty} \exp \left(-\frac{1}{2}\left(g\left(x_{i}, o\right)+g\left(y_{i}, o\right)-g\left(x_{i}, y_{i}\right)\right)\right)
\end{aligned}
$$




$$
\begin{aligned}
= & \lim _{i \rightarrow \infty} \exp \left(-\log \left(\frac{d_{\mathrm{CC}}\left(\pi\left(x_{i}\right), \pi(o)\right)+\max \left(h\left(x_{i}\right), h(o)\right)}{\sqrt{h\left(x_{i}\right) h(o)}}\right)\right. \\
& -\log \left(\frac{d_{\mathrm{CC}}\left(\pi\left(y_{i}\right), \pi(o)\right)+\max \left(h\left(y_{i}\right), h(o)\right)}{\sqrt{h\left(y_{i}\right) h(o)}}\right) \\
& \left.+\log \left(\frac{d_{\mathrm{CC}}\left(\pi\left(x_{i}\right), \pi\left(y_{i}\right)\right)+\max \left(h\left(x_{i}\right), h\left(y_{i}\right)\right)}{\sqrt{h\left(x_{i}\right) h\left(y_{i}\right)}}\right)\right) \\
= & \lim _{i \rightarrow \infty}\left(\frac{d_{\mathrm{CC}}(p, \pi(o))+h(o)}{\sqrt{h\left(x_{i}\right) h(o)}}\right)^{-1}\left(\frac{d_{\mathrm{CC}}(q, \pi(o))+h(o)}{d_{\mathrm{CC}}(p, q) h(o)}\right)^{-1} \frac{d_{\mathrm{CC}}(p, q)}{\sqrt{h\left(y_{i}\right) h(o)}} \\
= & \frac{\sqrt{h\left(x_{i}\right)}}{\left(d_{\mathrm{CC}}(p, \pi(o))+h(o)\right)\left(d_{\mathrm{CC}}(q, \pi(o))+h(o)\right)} .
\end{aligned}
$$

\section{Comparing $d$ and $g$, after Balogh and Bonk}

The quantitative bounds on the distortion of the identity map in Proposition 1.5 follow from the following result, which is a refinement of an analogue statement of Balogh and Bonk [3, Corollary 1.3]. We follow largely their arguments, but where in [3] the noise due to the rough geometry would yield an additive constant, here instead we need to exploit the fact that the geometry is asymptotically hyperbolic to show that such constants can be chosen arbitrarily small the closer one gets to the boundary.

Theorem 4.1 For every $\bar{p} \in \partial D$ and $\epsilon>0$ there exists $r>0$ such that for all distinct $p, q \in \partial D \cap B(\bar{p}, r)$ there exists $r^{\prime}>0$ such that for all $x \in D \cap B\left(p, r^{\prime}\right)$ and all $y \in D \cap B\left(q, r^{\prime}\right)$

$$
g(x, y)-\epsilon \leq d_{K}(x, y) \leq g(x, y)+\epsilon .
$$

In the rest of the paper we will refer to this result in connection with the quintuplet $(\bar{p}, p, q, x, y)$.

\subsection{Lemmata}

The proof of Theorem 4.1 is based on preliminary estimates established in Lemmas 4.3, 4.8, and 4.12.

Lemma 4.3 Let $\delta_{0}>0$ to be the constant in Lemma 2.5. For $x_{1}, x_{2} \in D$ with $d_{E}\left(x_{i}, \partial D\right)<\delta_{0}$, and $h\left(x_{1}\right) \geq h\left(x_{2}\right)$, consider a piecewise $C^{1}$ curve $\gamma:[0,1] \rightarrow N_{\delta_{0}}$ with $\gamma(0)=x_{1}$ and $\gamma(1)=x_{2}$. The length $l_{K}(\gamma)$ of $\gamma$ with respect to the metric $d_{K}$ satisfies

$$
l_{K}(\gamma) \geq \ln \frac{h\left(x_{1}\right)}{h\left(x_{2}\right)}-C\left(h\left(x_{1}\right)-h\left(x_{2}\right)\right),
$$

where $C$ is the same constant as in (2.8). Moreover, if the curve is a segment $\gamma(t)=$ $x_{1}+t\left(x_{2}-p_{1}\right) \subset \pi^{-1}(p)$ for some $p \in \partial D$ then one has

$$
l_{K}(\gamma) \leq \ln \frac{h\left(x_{1}\right)}{h\left(x_{2}\right)}+C\left(h\left(x_{1}\right)-h\left(x_{2}\right)\right),
$$


Proof Recall from [3, page 517] that

$$
\left|\frac{d}{d t} h(\gamma(t))\right|=\frac{\left|\operatorname{Re}\left\langle n(\pi(\gamma(t))), \gamma^{\prime}(t)\right\rangle\right|}{2 h(\gamma(t))} \leq \frac{\left|\gamma_{N}^{\prime}(t)\right|}{2 h(\gamma(t))} .
$$

The latter and (2.8) yield

$$
\begin{aligned}
l_{K}(\gamma) & =\int_{0}^{1} K\left(\gamma(t), \gamma^{\prime}(t)\right) d t \\
& \geq \int_{0}^{1}\left(1-C d_{E}^{\frac{1}{2}}(\gamma(t), \partial D)\right)\left(\frac{\left|\gamma_{N}^{\prime}(t)\right|^{2}}{4 d_{E}^{2}(\gamma(t), \partial D)}+(1-\bar{\epsilon}) \frac{L_{\varphi}\left(\pi\left(\gamma(t), \gamma_{H}^{\prime}(t)\right)\right.}{d_{E}(\gamma(t), \partial D)}\right)^{\frac{1}{2}} d t \\
& \geq \int_{0}^{1}\left(1-C d_{E}^{\frac{1}{2}}(\gamma(t), \partial D)\right) \frac{\left|\gamma_{N}^{\prime}(t)\right|}{2 d_{E}(\gamma(t), \partial D)} d t \geq \int_{0}^{1} \frac{(1-C h(\gamma(t))}{h(\gamma(t))} \frac{d}{d t} h(\gamma(t)) d t \\
& =\ln \frac{h\left(x_{1}\right)}{h\left(x_{2}\right)}-C\left(h\left(x_{1}\right)-h\left(x_{2}\right)\right),
\end{aligned}
$$

which gives (4.4).

On the other hand, if $\gamma(t)=x_{1}+t\left(x_{2}-x_{1}\right)$, then we observe that $\gamma^{\prime}$ is parallel to the unit normal at $\pi\left(x_{i}\right)$ and so has no tangent component, hence no horizontal component with respect to the splitting at $\pi\left(x_{i}\right)$. Using the fact that

$$
d_{E}(\gamma(t), \partial D)=|\gamma(t)-p|=\left|t\left(x_{2}-x_{1}\right)\right|+\left|x_{1}-p\right|
$$

and (2.8) one has

$$
\begin{aligned}
l_{K}(\gamma) & =\int_{0}^{1} K\left(\gamma(t), \gamma^{\prime}(t)\right) d t \\
& \leq \int_{0}^{1}\left(1+C d_{E}^{\frac{1}{2}}(\gamma(t), \partial D)\right)\left(\frac{\left|\gamma_{N}^{\prime}(t)\right|^{2}}{4 d_{E}^{2}(\gamma(t), \partial D)}+(1+\bar{\epsilon}) \frac{L_{\varphi}\left(\pi\left(\gamma(t), \gamma_{H}^{\prime}(t)\right)\right.}{d_{E}(\gamma(t), \partial D)}\right)^{\frac{1}{2}} d t \\
& =\frac{1}{2} \int_{0}^{1} \frac{\left|x_{2}-x_{1}\right|}{t\left|x_{2}-x_{1}\right|+\left|x_{1}-p\right|} d t+\frac{C}{2} \int_{0}^{1} \frac{1}{\sqrt{t\left|x_{2}-x_{1}\right|+\left|x_{1}-p\right|}} d t \\
& =\ln \frac{h\left(x_{1}\right)}{h\left(x_{2}\right)}+C\left(h\left(x_{1}\right)-h\left(x_{2}\right)\right),
\end{aligned}
$$

which gives (4.5).

An immediate consequence of Lemma 4.3 is the following.

Corollary 4.6 Let $\delta_{0}>0$ to be the constant in Proposition 2.5. If $x_{1}, x_{2} \in D$, with $\delta_{0}>h\left(x_{1}\right) \geq h\left(x_{2}\right)$, then

$$
\ln \frac{h\left(x_{1}\right)}{h\left(x_{2}\right)}-C\left(h\left(x_{1}\right)-h\left(x_{2}\right)\right) \leq d_{K}\left(x_{1}, x_{2}\right) .
$$


Moreover, if $\pi\left(x_{1}\right)=\pi\left(p_{2}\right)$, then we also have

$$
d_{K}\left(x_{1}, x_{2}\right) \leq \ln \frac{h\left(x_{1}\right)}{h\left(x_{2}\right)}+C\left(h\left(x_{1}\right)-h\left(x_{2}\right)\right)
$$

where $C$ is the same constant as in (2.8).

The next lemma provides an upper bound for $d_{K}\left(x_{1}, x_{2}\right)$ in the case when both points $x_{1}, x_{2}$ are at the same distance from the boundary and equal to the CarnotCarathéodory distance between the projections $\pi\left(x_{1}\right), \pi\left(x_{2}\right)$.

Lemma 4.8 Let $p_{1}, p_{2} \in \partial D$. If we set $x_{i}:=p_{i}-d_{C C}\left(p_{1}, p_{2}\right) n\left(p_{i}\right), i=1,2$, then

$$
d_{K}\left(x_{1}, x_{2}\right) \rightarrow 0, \quad \text { as } d_{C C}\left(p_{1}, p_{2}\right) \rightarrow 0
$$

Proof Let $\eta>0$ and let $\alpha:[0,1] \rightarrow \partial D$ be any horizontal curve with $\alpha(0)=p_{1}$ and $\alpha(1)=p_{2}$, such that its subRiemannian length $l_{C C}$, satisfies

$$
l_{C C}(\alpha)=\int_{0}^{1} L_{\varphi}^{\frac{1}{2}}\left(\alpha(t), \alpha^{\prime}(t)\right) d t \leq d_{C C}\left(p_{1}, p_{2}\right)(1+\eta) .
$$

Define a new curve $\gamma:[0,1] \rightarrow D$ as a lift at height $h \in\left(0, \delta_{0}\right)$ of $\alpha$ by the formula

$$
\gamma(t):=\alpha(t)-h n(\alpha(t)) .
$$

Arguing as in the proof of [3, Lemma 2.2] yields the following relations between $\alpha^{\prime}$ and $\gamma^{\prime}$,

$$
\begin{aligned}
L\left(\alpha(t), \gamma_{H}^{\prime}(t)\right) & =L\left(\alpha(t), \alpha^{\prime}(t)\right)+O\left(h\left|\alpha^{\prime}(t)\right|^{2}\right) \\
\left|\gamma_{N}^{\prime}(t)\right| & =O\left(h\left|\alpha^{\prime}(t)\right|\right) .
\end{aligned}
$$

In fact, from (4.10) one has $\left.\gamma^{\prime}(t)\right|_{H}=\alpha^{\prime}(t)-h\left[\left.d n\right|_{\alpha(t)} \alpha^{\prime}(t)\right]_{H}$ which, together with the bilinearity of the Levi form, yields (4.11). Consequently we have

$$
\begin{aligned}
l_{K}(\gamma) & =\int_{0}^{1} K\left(\gamma(t), \gamma^{\prime}(t)\right) d t \\
& \leq \int_{0}^{1}\left(1+C d_{E}^{\frac{1}{2}}(\gamma(t), \partial D)\right)\left(\frac{\left|\gamma_{N}^{\prime}(t)\right|^{2}}{4 d_{E}^{2}(\gamma(t), \partial D)}+(1+\bar{\epsilon}) \frac{L_{\varphi}\left(\pi\left(\gamma(t), \gamma_{H}^{\prime}(t)\right)\right.}{d_{E}(\gamma(t), \partial D)}\right)^{\frac{1}{2}} d t \\
& =\int_{0}^{1}\left(1+C h^{\frac{1}{2}}\right)\left(\frac{\left|\gamma_{N}^{\prime}(t)\right|^{2}}{4 h^{2}}+(1+\bar{\epsilon}) \frac{L_{\varphi}\left(\pi\left(\gamma(t), \gamma_{H}^{\prime}(t)\right)\right.}{h}\right)^{\frac{1}{2}} d t \\
& \leq \int_{0}^{1}\left(1+C h^{\frac{1}{2}}\right)\left(C\left|\alpha^{\prime}(t)\right|^{2}+(1+\bar{\epsilon}) \frac{L_{\varphi}\left(\alpha(t), \alpha^{\prime}(t)\right)}{h}\right)^{\frac{1}{2}} d t \\
& \leq(1+C \sqrt{h})(1+\eta)\left[C d_{C C}\left(p_{1}, p_{2}\right)+(1+\bar{\epsilon})^{\frac{1}{2}} h^{-\frac{1}{2}} d_{C C}\left(p_{1}, p_{2}\right)\right] .
\end{aligned}
$$

Setting $h=d_{C C}\left(x_{1}, x_{2}\right)$ in the latter yields the conclusion. 
The next lemma will be instrumental in establishing a lower bound for $d_{K}\left(x_{1}, x_{2}\right)$ in the case when a length minimizing arc $\gamma$ joining two points $x_{1}, x_{2} \in D$ will travel at a distance further than the Carnot-Carathéodory distance between their projections.

Lemma 4.12 Let $\delta_{0}>0$ be smaller than the similarly named constants in Propositions 2.5 and 2.7. Consider two points $x_{1}, x_{2} \in D$ with $d_{E}\left(x_{i}, \partial D\right)<\delta_{0}$. Set $p_{i}=\pi\left(x_{i}\right) \in \partial D$, and let $\gamma:[0,1] \rightarrow D$ denote an arc joining $x_{1}$ and $x_{2}$. If $\max _{z \in \gamma} h(z) \geq d_{C C}\left(p_{1}, p_{2}\right)$ then

$$
l_{K}(\gamma) \geq 2 \ln \left(\frac{d_{C C}\left(p_{1}, p_{2}\right)}{\sqrt{h\left(x_{1}\right) h\left(x_{2}\right)}}\right)-C\left(2 d_{C C}\left(p_{1}, p_{2}\right)-h\left(x_{1}\right)-h\left(x_{2}\right)\right),
$$

where $C$ is the same constant as in (2.8).

Proof Choose $t_{0} \in[0,1]$ such that $h\left(\gamma\left(t_{0}\right)\right)=\max _{z \in \gamma} h(z)$. Set $\gamma_{1}, \gamma_{2}$ be the two branches of the curve $\gamma$ corresponding to the subintervals $\left[0, t_{0}\right]$ and $\left[t_{0}, 1\right]$. Set also $\bar{\gamma}_{1}$ and $\bar{\gamma}_{2}$ to be the connected components on $\gamma_{1}$ and $\gamma_{2}$ joining $x_{i}$ to the closest points $y_{i} \in \gamma$ such that $h\left(y_{i}\right)=d_{C C}\left(p_{1}, p_{2}\right)$, for $i=1,2$. More formally, $y_{1}=\gamma\left(t_{1}\right)$, with $t_{1}=\inf \left\{t \in\left[0, t_{1}\right]\right.$ such that $\left.h(\gamma(t)) \geq d_{C C}\left(p_{1}, p_{2}\right)\right\}$. The point $y_{2}$ is defined analogously.

Next we invoke Lemma 4.3 to deduce

$$
\begin{aligned}
l_{K}(\gamma) & \geq l_{K}\left(\bar{\gamma}_{1}\right)+l_{K}\left(\bar{\gamma}_{2}\right) \\
& \geq \ln \frac{d_{C C}\left(p_{1}, p_{2}\right)}{h\left(x_{1}\right)}+\ln \frac{d_{C C}\left(p_{1}, p_{2}\right)}{h\left(x_{2}\right)}-C\left(2 d_{C C}\left(p_{1}, p_{2}\right)-h\left(x_{1}\right)-h\left(x_{2}\right)\right) \\
& =2 \ln \left(\frac{d_{C C}\left(p_{1}, p_{2}\right)}{\sqrt{h\left(x_{1}\right) h\left(x_{2}\right)}}\right)-C\left(2 d_{C C}\left(x_{1}, x_{2}\right)-h\left(x_{1}\right)-h\left(x_{2}\right)\right),
\end{aligned}
$$

which is the desired bound (4.13).

\subsection{Proof of Theorem 4.1}

Thanks to the previous lemmata we can now prove the main result of the section.

Proof of Theorem 4.1 We shall show that for all $\bar{p} \in \partial D$ and $\epsilon>0$ one can choose $r>0$ small enough so that for all distinct $p, q \in \partial D \cap B(\bar{p}, r)$ one can find $r^{\prime} \in(0, r)$ such that (4.2) holds for all $x \in D \cap B\left(p, r^{\prime}\right)$ and all $y \in D \cap B\left(q, r^{\prime}\right)$. In our proof we begin with arbitrary values of $r$ and $r^{\prime}$ and then put several constrains on them.

If $p$ and $q$ are distinct, then the value $d_{1}:=d_{C C}(p, q)$ is strictly positive. We shall choose $r$ smaller that the constants $\delta_{0}$ in Propositions 2.5 and 2.7 and so that $d_{1}$ is small enough to be determined later. Denote by $\bar{x}$, and $\bar{y}$ the projections on the boundary of $x$ and $y$, respectively. Note that since the projections are the closest points in $\partial D$, then $\bar{x} \in B\left(p, 2 r^{\prime}\right)$ and $\bar{y} \in B\left(q, 2 r^{\prime}\right)$. Set $d_{2}:=d_{C C}(\bar{x}, \bar{y})$. Notice that as $r^{\prime} \rightarrow 0$ we have $d_{2} \rightarrow d_{1}$. We shall choose $r^{\prime}$ sufficiently small so that $r^{\prime}<d_{2}$ and $d_{2} \in\left(d_{1} / 2,2 d_{1}\right)$. In particular, if $r$ was chosen small enough, then $d_{2}$ is positive and smaller than the constants $\delta_{0}$ in Propositions 2.5 and 2.7. 
Proof of the upper bound in (4.2). Set $x^{\prime}:=\bar{x}-d_{2} n(\bar{x})$ and $y^{\prime}:=\bar{y}-d_{2} n(\bar{y})$, so $x^{\prime}$, $y^{\prime}$ are points in $D$ at distance $d_{2}$ from $\partial D$ and with the same projection on $\partial D$ as $x$, $y$, respectively.

By Lemma 4.8 we can choose $d_{1}$ sufficiently small so that $d_{K}\left(x^{\prime}, y^{\prime}\right)<\epsilon / 3$. Invoking (4.7), since $h\left(x^{\prime}\right)=h\left(y^{\prime}\right)=d_{2}>\max \{h(x), h(y)\}$, yields

$$
\begin{aligned}
& d_{K}\left(x, x^{\prime}\right) \leq \log \left(d_{2} / h(x)\right)+C\left(d_{2}-h(x)\right) \quad \text { and } \\
& d_{K}\left(y, y^{\prime}\right) \leq \log \left(d_{2} / h(y)\right)+C\left(d_{2}-h(y)\right) .
\end{aligned}
$$

Choose $d_{1}$ chosen sufficiently small so that $C d_{2} \leq \epsilon / 3$.

Combining the previous bounds with the definition of $g$, we obtain the following estimates

$$
\begin{aligned}
d_{K}(x, y)-g(x, y) \leq & d_{K}\left(x, x^{\prime}\right)+d_{K}\left(x^{\prime}, y^{\prime}\right)+d_{K}\left(y^{\prime}, y\right)-g(x, y) \\
\leq & \log \left(d_{2} / h(x)\right)+C\left(d_{2}-h(x)\right)+\epsilon / 3 \\
& +\log \left(d_{2} / h(y)\right)+C\left(d_{2}-h(y)\right)-2 \ln \left(\frac{\left.d_{2}+h(x) \wedge h(y)\right)}{\sqrt{h(x) h(y)}}\right) \\
\leq & \epsilon-C h(x)-C h(y)-2 \ln \left(1+\frac{h(x) \wedge h(y)}{d_{2}}\right)<\epsilon,
\end{aligned}
$$

where we used that the terms $h(x), h(y), \ln \left(1+\frac{h(x) \wedge h(y)}{d_{2}}\right)$ are positive. This conclude the proof of the upper bound in (4.2).

Proof of the lower bound in (4.2). Choose $\delta>0$ such that $\ln (1 /(1+\delta))<\epsilon$ and $r^{\prime}>0$ small enough so that $\frac{\max (h(x), h(y))}{d_{C C}(p, q)} \leq \delta$, for all $x \in D \cap B\left(p, r^{\prime}\right)$ and all $y \in D \cap B\left(q, r^{\prime}\right)$. Consider any $\operatorname{arc} \gamma:[0,1] \rightarrow D$ joining $x$ and $y$, and set $H:=$ $\max _{z \in \gamma} h(z)$.

- If $H \geq d_{C C}(\bar{x}, \bar{y})$ then in view of Lemma 4.12 we have

$$
\begin{aligned}
d_{K}(x, y)-g(x, y) \geq & \left.2 \ln \left(\frac{d_{2}}{\sqrt{h(x) h(y)}}\right)-C\left(2 d_{2}-h(x)-h(y)\right)\right) \\
& -2 \ln \left(\frac{\left.d_{2}+h(x) \wedge h(y)\right)}{\sqrt{h(x) h(y)}}\right) \\
\geq & 2 \ln \left(\frac{1}{1+\frac{\max (h(x), h(y))}{d_{2}}}\right)-C\left(2 d_{2}-h(x)-h(y)\right) \\
\geq & -(C+2) \epsilon .
\end{aligned}
$$

In this case the proof is concluded.

- If $H \leq d_{C C}(\bar{x}, \bar{y})$ then it follows that $H$ is smaller than the constants $\delta_{0}$ in Propositions 2.5 and 2.7. In particular we can assume without loss of generality that $C H<1 / 2$, where $C$ is as in (2.8). Let $t_{0} \in[0,1]$ be such that $h\left(\gamma\left(t_{0}\right)\right)=H$ and consider the two branches $\gamma_{1}, \gamma_{2}$ of $\gamma$ given by restrictions to $\left[0, t_{0}\right]$ and $\left[t_{0}, 1\right]$. Given $\epsilon>0$ as in the statement, let $\theta \in(1,2]$ so that $\ln \theta<\epsilon$ and define $k \in \mathbb{N}$ such that 


$$
h(\gamma(0)) \in\left[\frac{H}{\theta^{k}}, \frac{H}{\theta^{k-1}}\right] .
$$

Following [3], we define $s_{0}, s_{1}, \ldots, s_{k} \in\left[0, t_{0}\right]$ such that $s_{0}=0$ and

$$
s_{l}=\min \left\{s \in\left[0, t_{0}\right] \text { such that } h(\gamma(s))=\frac{H}{\theta^{k-l}}\right\} .
$$

Set $t_{1}=s_{k} \leq t_{0}$ and for each $l=1, \ldots, k$,

$$
v_{l}^{-1}=\frac{d_{C C}(\bar{x}, \bar{y}) \cdot(\theta-1)}{8 \theta^{k-l}} .
$$

For each of the two branches $\gamma_{1}, \gamma_{2}$, we distinguish two alternatives:

- Alternative \#1 (All sub-arcs have large slope) In this alternative we assume that for every $l=1, \ldots, k$ one has

$$
d_{C C}\left(\pi\left(\gamma\left(s_{l-1}\right)\right), \pi\left(\gamma\left(s_{l}\right)\right)\right) \leq v_{l}^{-1}
$$

From the latter we draw two conclusions. The first is a simple application of the triangle inequality,

$$
\begin{aligned}
& d_{C C}\left(\bar{z}, \pi\left(\gamma\left(t_{1}\right)\right)\right. \\
& \leq \sum_{l=1}^{k} d_{C C}\left(\pi\left(\gamma\left(s_{l-1}\right)\right), \pi\left(\gamma\left(s_{l}\right)\right)\right) \leq(\theta-1) \frac{d_{C C}(\bar{x}, \bar{y})}{8 \theta^{k}} \sum_{l=1}^{k} \theta^{l} \leq \frac{d_{C C}(\bar{x}, \bar{y})}{4} .
\end{aligned}
$$

On the other hand, in view of Lemma 4.3 one has

$$
l_{K}\left(\left.\gamma\right|_{\left[0, t_{1}\right]}\right) \geq \ln \frac{h\left(\gamma\left(t_{1}\right)\right)}{h(x)}-C\left(h\left(\gamma\left(t_{1}\right)\right)-h(x)\right)=\ln \frac{H}{h(x)}-C(H-h(x)) .
$$

- Alternative \#2 (One sub-arc has small slope) In this alternative, we assume that there exists $l \in\{1, \ldots, k\}$ such that

$$
d_{C C}\left(\pi\left(\gamma\left(s_{l-1}\right)\right), \pi\left(\gamma\left(s_{l}\right)\right)\right)>v_{l}^{-1}
$$

Note that if $s \in\left[s_{l-1}, s_{l}\right]$ then from the definition of the points $s_{l}$, one has

$$
h(\gamma(s)) \leq \theta^{l-k} H \leq \frac{8}{\theta-1} v_{l}^{-1} .
$$

We then claim that there exists a constant $\mathcal{C}>0$ depending only on the defining function $\varphi$ such that

$$
l_{K}\left(\left.\gamma\right|_{\left[s_{l-1}, s_{l}\right]}\right) \geq \mathcal{C}(\theta-1)^{2} \frac{d_{C C}(\bar{x}, \bar{y})}{H} .
$$


Indeed, arguing as in [3, page 521] we invoke (2.8), Lemma 2.10, and (4.16) and we bound as follows:

$$
\begin{aligned}
l_{K}\left(\left.\gamma\right|_{\left[s_{l-1}, s_{l}\right]}\right) \\
\geq \\
\geq \mathcal{C} \frac{(1-C H) \theta^{k-l}}{H} \int_{s_{l-1}}^{s_{l}}\left[L_{\varphi}\left(\pi(\gamma(s)),[\pi(\gamma(s))]_{H}^{\prime}\right)\right. \\
\left.\quad+(\theta-1)^{2} v_{l}^{2}\left|[\pi(\gamma(s))]_{N}^{\prime}\right|^{2}\right]^{\frac{1}{2}} d s \\
\geq \\
\quad \frac{\mathcal{C}}{2}(\theta-1) \frac{\theta^{k-l}}{H} \int_{s_{l-1}}^{s_{l}}\left[L_{\varphi}\left(\pi(\gamma(s)),[\pi(\gamma(s))]_{H}^{\prime}\right)+v_{l}^{2}\left|[\pi(\gamma(s))]_{N}^{\prime}\right|^{2}\right]^{\frac{1}{2}} d s \\
\geq \mathcal{C}(\theta-1) \frac{\theta^{k-l}}{H} d_{\nu_{l}}\left(\pi\left(\gamma\left(s_{l-1}\right)\right), \pi\left(\gamma\left(s_{l}\right)\right)\right) \\
\geq \mathcal{C}(\theta-1) \frac{\theta^{k-l}}{H} d_{C C}\left(\pi\left(\gamma\left(s_{l-1}\right)\right), \pi\left(\gamma\left(s_{l}\right)\right)\right) \\
\geq \mathcal{C}(\theta-1)^{2} \frac{d_{C C}(\bar{x}, \bar{y})}{H}
\end{aligned}
$$

where $d_{v_{l}}$ denotes the approximation of the Carnot-Carathéodory metric defined in (2.9).

Next we claim that

$$
l_{L}\left(\left.\gamma\right|_{\left[0, t_{1}\right]}\right) \geq \ln \left(\frac{H}{h(y)}\right)+\frac{\mathcal{C}(\theta-1)^{2}}{H} d_{C C}(\bar{x}, \bar{y})-C(H-h(y))-\epsilon .
$$

Indeed, Lemma 4.3 and (4.17) yields

$$
\begin{aligned}
l_{L}\left(\left.\gamma\right|_{\left[0, t_{1}\right]}\right)= & l_{K}\left(\left.\gamma\right|_{\left[0, s_{l-1}\right]}\right)+l_{K}\left(\left.\gamma\right|_{\left[s_{l-1}, s_{l}\right]}\right)+l_{K}\left(\left.\gamma\right|_{\left[s_{l}, t_{l}\right]}\right) \\
\geq & \ln \left(\frac{H}{h\left(\gamma\left(s_{l}\right)\right)} \frac{h\left(\gamma\left(s_{l-1}\right)\right)}{h(x)}\right)+\frac{\mathcal{C}(\theta-1)^{2}}{H} d_{C C}(\bar{x}, \bar{y}) \\
& -C\left(H-h\left(\gamma\left(s_{l}\right)+h\left(\gamma\left(s_{l-1}\right)\right)-h(x)\right)\right. \\
\geq & \ln \left(\frac{H}{h(x)} \theta^{-1}\right)+\frac{\mathcal{C}(\theta-1)^{2}}{H} d_{C C}(\bar{x}, \bar{y})-C(H-h(x)) \\
\geq & \ln \left(\frac{H}{h(x)}\right)+\frac{\mathcal{C}(\theta-1)^{2}}{H} d_{C C}(\bar{x}, \bar{y})-C(H-h(x))-\epsilon .
\end{aligned}
$$

Applying similar consideration to the branch $\gamma_{2}$ one obtains a $t_{2} \in\left[t_{0}, 1\right]$ such that one of the following two alternatives hold: Either

$$
d_{C C}\left(\bar{y}, \pi\left(\gamma\left(t_{2}\right)\right) \leq \frac{d_{C C}(\bar{x}, \bar{y})}{4} \text { and } l_{K}\left(\left.\gamma\right|_{\left[t_{2}, 1\right]}\right) \geq \ln \frac{H}{h(y)}-C(H-h(y)) .\right.
$$


or

$$
l_{L}\left(\left.\gamma\right|_{\left[t_{2}, 1\right]}\right) \geq \ln \left(\frac{H}{h(y)}\right)+\frac{\mathcal{C}(\theta-1)^{2}}{H} d_{C C}(\bar{x}, \bar{y})-C(H-h(y))-\epsilon .
$$

To conclude the proof we need to examine all possible combinations of these alternatives. We will show that in each case one obtains

$$
l_{K}(\gamma) \geq 2 \ln \left(\frac{d_{C C}(\bar{x}, \bar{y})}{\sqrt{h(x) h(y)}}\right)-C\left(2 d_{C C}(\bar{x}, \bar{y})-h(x)-h(y)\right)-\epsilon .
$$

- Suppose both (A1) and (B1) hold. Observe that

$$
\begin{aligned}
d_{C C}\left(\pi\left(\gamma\left(t_{1}\right)\right), \pi\left(\gamma\left(t_{2}\right)\right)\right) & \geq d_{C C}(\bar{x}, \bar{y})-d_{C C}\left(\bar{x}, \pi\left(\gamma\left(t_{1}\right)\right)\right)-d_{C C}\left(\bar{y}, \pi\left(\gamma\left(t_{2}\right)\right)\right) \\
& \geq \frac{d_{C C}(\bar{x}, \bar{y})}{2} .
\end{aligned}
$$

Repeating the argument in (4.17) for $l=k$ and invoking the Riemannian approximation lemma [3, Lemma 3.2] one has

$$
l_{L}\left(\left.\gamma\right|_{\left[t_{1}, t_{2}\right]}\right) \geq \mathcal{C}(\theta-1)^{2} \frac{d_{\nu_{k}}\left(\pi\left(\gamma\left(t_{1}\right)\right), \pi\left(\gamma\left(t_{2}\right)\right)\right)}{H} \geq \mathcal{C}(\theta-1)^{2} \frac{d_{C C}(\bar{x}, \bar{y})}{H} .
$$

The latter, together with (A1 (ii)), and the second inequality in (B1) yields

$$
l_{K}(\gamma) \geq 2 \ln \left(\frac{H}{\sqrt{h(x) h(y)}}\right)+\mathcal{C}(\theta-1)^{2} \frac{d_{C C}(\bar{x}, \bar{y})}{H}-C(2 H-h(x)-h(y)) .
$$

Since the right hand side is monotone decreasing in $H \leq d_{C C}(\bar{x}, \bar{y})$ then one has

$$
\begin{aligned}
l_{K}(\gamma) & \geq 2 \ln \left(\frac{d_{C C}(\bar{x}, \bar{y})}{\sqrt{h(x) h(y)}}\right)+\mathcal{C}(\theta-1)^{2}-C\left(2 d_{C C}(\bar{x}, \bar{y})-h(x)-h(y)\right) \\
& \geq 2 \ln \left(\frac{d_{C C}(\bar{x}, \bar{y})}{\sqrt{h(x) h(y)}}\right)-C\left(2 d_{C C}(\bar{x}, \bar{y})-h(x)-h(y)\right)
\end{aligned}
$$

completing the proof of (4.18).

- Suppose both (A1) and (B2) hold. One immediately has

$$
\begin{aligned}
l_{K}(\gamma) \geq & l_{K}\left(\gamma_{\left[0, t_{1}\right]}\right)+l_{K}\left(\left.\gamma\right|_{\left[t_{2}, 1\right]}\right) \\
\geq & \ln \left(\frac{H}{h(x)}\right)+\frac{\mathcal{C}(\theta-1)}{H} d_{C C}(\bar{x}, \bar{y})-C[H-h(x)] \\
& \quad-\epsilon+\ln \frac{H}{h(y)}-C(H-h(y)) .
\end{aligned}
$$

Applying the same consideration as above we immediately deduce (4.18). 
- Suppose both (A2) and (B1) hold. This combination is dealt with analogously to the previous case.

- Suppose both (A2) and (B2) hold. Estimate (4.18) follows immediately from (A2) and (B2).

To conclude the proof we need to consider the infimum of $l_{K}(\gamma)$ among all arcs $\gamma$ joining $x$ and $y$ and apply (4.18) to each. One has

$$
\begin{gathered}
d_{K}(x, y)-g(x, y) \geq 2 \ln \left(\frac{d_{C C}(\bar{x}, \bar{y})}{\sqrt{h(x) h(y)}} \frac{1}{\frac{d_{C C}(\bar{x}, \bar{y})}{\sqrt{h(x) h(y)}}+\frac{\max \{h(x), h(y)\}}{\sqrt{h(x) h(y)}}}\right) \\
-C\left(2 d_{C C}(\bar{x}, \bar{y})-h(x)-h(y)\right)-\epsilon \\
=-2 \ln \left(1+\frac{\max \{h(x), h(y)\}}{d_{C C}(\bar{x}, \bar{y})}\right) \\
-C\left(2 d_{C C}(\bar{x}, \bar{y})-h(x)-h(y)\right)-\epsilon .
\end{gathered}
$$

The proof is then concluded by applying the same argument as in (4.14).

\section{Local biLipschitz equivalence of visual quasi-distances and proof of main result}

In this section we prove Proposition 1.5 and the main result, Theorem 1.1.

Proof of Proposition 1.5 Let $\bar{p}$ as in the statement and choose $\epsilon>0$ such that $\exp \left(\frac{3}{2} \epsilon\right) \leq 1+\bar{\epsilon}$. Invoke Theorem 4.1 in correspondence to the choice of $\bar{p}$ and $\epsilon$, to obtain the value $r>0$ and select any $\omega \in \partial D \cap B(\bar{p}, r) \backslash\{\bar{p}\}$. In correspondence to this choice of $\omega$, Theorem 4.1 yields a smaller radius $0<r^{\prime}<r$, so that if we choose $y \in D \cap B\left(\bar{p}, r^{\prime}\right)$ and $o \in D \cap B\left(\omega, r^{\prime}\right)$ and then apply Theorem 4.1 to the quintuplet $(\bar{p}, \bar{p}, \omega, y, o)$ we obtain

$$
\left|g(y, o)-d_{K}(y, o)\right|<\epsilon, \quad \text { for all } y \in D \cap B\left(\bar{p}, r^{\prime}\right), \text { and } o \in D \cap B\left(\omega, r^{\prime}\right)
$$

Next, given $p, q \in \partial D \cap B\left(\bar{p}, r^{\prime}\right)$ we similarly use Theorem 4.1 to infer the existence of a $r^{\prime \prime}>0$ for which, applying Theorem 4.1 to the quintuplet $(\bar{p}, p, q x, y)$

$\left|d_{K}(x, y)-g(x, y)\right| \leq \epsilon, \quad$ for all $x \in D \cap B\left(p, r^{\prime \prime}\right)$, and for all $y \in D \cap B\left(q, r^{\prime \prime}\right)$.

If $x_{i}$ (resp., $y_{i}$ ) is a sequence in $D$ converging to $p$ (resp., $q$ ), then for $i$ large enough $x_{i} \in D \cap B\left(p, r^{\prime \prime}\right)$ and $y_{i} \in D \cap B\left(q, r^{\prime \prime}\right)$ and $x_{i}, y_{i} \in B\left(\bar{p}, r^{\prime}\right)$. From the above bounds one obtains

$$
\begin{aligned}
\left|\left\langle y_{i}, x_{i}\right\rangle_{o}^{g}-\left\langle y_{i}, x_{i}\right\rangle_{o}^{K}\right|= & \frac{1}{2} \mid g\left(y_{i}, o\right)-d_{K}\left(y_{i}, o\right)+g\left(x_{i}, o\right) \\
& -d_{K}\left(x_{i}, o\right)+d_{K}\left(x_{i}, y_{i}\right)-g\left(x_{i}, y_{i}\right) \mid \\
\leq & \frac{3}{2} \epsilon .
\end{aligned}
$$


Consequently, if the sequences $x_{i}, y_{i}$ are taken so that $\langle p, q\rangle_{o}^{g}=\lim _{i \rightarrow \infty}\left\langle y_{i}, x_{i}\right\rangle_{o}^{g}$, we have

$$
\begin{aligned}
\frac{\rho_{o}^{K}(p, q)}{\rho_{o}^{g}(p, q)} & \leq \frac{\lim _{i \rightarrow \infty} \exp \left(-\left\langle y_{i}, x_{i}\right\rangle_{o}^{K}\right)}{\lim _{i \rightarrow \infty} \exp \left(-\left\langle y_{i}, x_{i}\right\rangle_{o}^{g}\right)} \\
& =\lim _{i \rightarrow \infty} \exp \left(\left\langle y_{i}, x_{i}\right\rangle_{o}^{g}-\left\langle y_{i}, x_{i}\right\rangle_{o}^{K}\right) \\
& \leq \exp \left(\frac{3}{2} \epsilon\right) \leq 1+\bar{\epsilon}
\end{aligned}
$$

And similarly, $\rho_{o}^{g}(p, q) / \rho_{o}^{K}(p, q)$ is bounded by $1+\bar{\epsilon}$.

Proof of Theorem 1.1 For any $\bar{p} \in \partial D_{1}$ and $\bar{\epsilon}>0$ we show that the boundary extension is $(1+\bar{\epsilon})$-quasi-conformal at $\bar{p}$, i.e. $H^{*}\left(\bar{p}, F, d_{C C}, d_{C C}\right) \leq 1+\bar{\epsilon}$, where $H^{*}$ is defined as in (2.1). Following the diagram (D) in the introduction, from (2.3) for every $o \in D_{1}$ we have

$$
\begin{aligned}
& H^{*}\left(\bar{p}, F, d_{C C}, d_{C C}\right) \\
& \quad \leq H^{*}\left(\bar{p}, \operatorname{Id}_{\partial D_{1}}, d_{\mathrm{CC}}, \rho_{o}^{g}\right) H^{*}\left(\bar{p}, \operatorname{Id}_{\partial D_{1}}, \rho_{o}^{g}, \rho_{o}^{K}\right) H^{*}\left(\bar{p}, F, \rho_{o}^{K}, \rho_{f(o)}^{K}\right) \\
& \quad \cdot H^{*}\left(F(\bar{p}), \operatorname{Id}_{\partial D_{2}}, \rho_{f(o)}^{K}, \rho_{f(o)}^{g}\right) H^{*}\left(F(\bar{p}), \operatorname{Id}_{\partial D_{2}}, \rho_{f(o)}^{g}, d_{\mathrm{CC}}\right) .
\end{aligned}
$$

Start by observing that for any $o \in D_{1}$ the pointed metric spaces $\left(D_{1}, d_{K}, o\right)$ and $\left(D_{2}, d_{K}, f(o)\right)$ are isometric. Thus they give rise to visual boundaries that are isometric with respect to the induced distances $\rho_{o}^{K}$ an $\rho_{f(o)}^{K}$, as defined in (1.3). Consequently the induced extension map $F:\left(\partial D_{1}, \rho_{o}^{K}\right) \rightarrow\left(\partial D_{2}, \rho_{f(o)}^{K}\right)$ is an isometry, and hence from (2.4)

$$
H^{*}\left(\bar{p}, F, \rho_{o}^{K}, \rho_{f(o)}^{K}\right)=1 .
$$

Regarding the first and last term in the right-hand side of (5.1), in view of Proposition 1.4 we have that

$$
H^{*}\left(\bar{p}, \operatorname{Id}_{\partial D_{1}}, d_{\mathrm{CC}}, \rho_{o}^{g}\right)=H^{*}\left(F(\bar{p}), \operatorname{Id}_{\partial D_{2}}, \rho_{f(o)}^{g}, d_{\mathrm{CC}}\right)=1 .
$$

We shall then prove that

$$
H^{*}\left(\bar{p}, \operatorname{Id}_{\partial D_{1}}, \rho_{o}^{g}, \rho_{o}^{K}\right) \leq 1+\bar{\epsilon} \text { and } H^{*}\left(F(\bar{p}), \operatorname{Id}_{\partial D_{2}}, \rho_{f(o)}^{K}, \rho_{f(o)}^{g}\right) \leq 1+\bar{\epsilon}
$$

for some suitable choice of $o$. To prove this we will need to invoke Proposition 1.5 twice, in $D_{1}$ and in $D_{2}$, together with the observation (2.4). Namely, we shall prove that for a suitable choice of $o$ The maps considered in (5.4) are $(1+\bar{\epsilon})$-biLipschitz in a neighborhood of the considered points.

First we apply Proposition 1.5 in a neighborhood of $F(\bar{p}) \in \partial D_{2}$, thus yielding $r_{2}>0$ such that for all $\omega_{2} \in \partial D_{2} \cap B\left(F(\bar{p}), r_{2}\right) \backslash\{F(\bar{p})\}$ there exists $r_{2}^{\prime}>0$ such that for all $o_{2} \in D_{2} \cap B\left(\omega_{2}, r_{2}^{\prime}\right)$ one has that $\rho_{o_{2}}^{g}$ and $\rho_{o_{2}}^{K}$ are $(1+\bar{\epsilon})$-biLipschitz in д $D_{2} \cap B\left(F(\bar{p}), r_{2}^{\prime}\right)$. For the moment we do not choose any specific $\omega_{2}$ and $o_{2}$, so $r_{2}^{\prime}$ is still to be determined. 
Next, we apply Proposition 1.5 to $D_{1}$ in a neighborhood of $\bar{p}$ and use it to choose $r_{1}>0$ such that for all $\omega_{1} \in \partial D_{1} \cap B\left(\bar{p}, r_{1}\right) \backslash\{\bar{p}\}$ there exists $r_{1}^{\prime}>0$ such that $o_{1} \in D_{1} \cap B\left(\omega_{1}, r_{1}^{\prime}\right)$ one has that $\rho_{o_{1}}^{g}$ and $\rho_{o_{1}}^{K}$ are $(1+\bar{\epsilon})$-biLipschitz in $\partial D_{1} \cap$ $B\left(\bar{p}, r_{1}^{\prime}\right)$. By continuity of the map $F$ we may have chosen $r_{1}$ small enough that $F\left(B\left(\bar{p}, r_{1}\right) \cap D_{1}\right) \subset B\left(F(\bar{p}), r_{2}\right) \cap D_{2}$.

We set $\omega_{2}:=F\left(\omega_{1}\right)$, which is then in $B\left(F(\bar{p}), r_{2}\right) \cap D_{2}$ and is different than $F(\bar{p})$ since $F$ is a homeomorphism. Now we fix $r_{2}^{\prime}$ accordingly, as we explained above. If needed we will select a smaller value for $r_{1}^{\prime}$ so that we can assume $F\left(B\left(\omega_{1}, r_{1}^{\prime}\right) \cap D_{1}\right) \subset$ $B\left(F\left(\omega_{1}\right), r_{2}^{\prime}\right) \cap D_{2}$.

To conclude, we can now select any base point $o \in B\left(\omega_{1}, r_{1}^{\prime}\right) \cap D_{1}$, so that $f(o) \in$ $B\left(\omega_{2}, r_{2}^{\prime}\right) \cap D_{2}$ and and hence $\rho_{o_{1}}^{g}$ and $\rho_{o_{1}}^{K}$ are $(1+\bar{\epsilon})$-biLipschitz in $\partial D_{1} \cap B\left(\bar{p}, r_{1}^{\prime}\right)$ and $\rho_{o_{2}}^{g}$ and $\rho_{O_{2}}^{K}$ are $(1+\bar{\epsilon})$-biLipschitz in $\partial D_{2} \cap B\left(F(\bar{p}), r_{2}^{\prime}\right)$. Thus, (2.4) gives (5.4).

Using the estimates (5.2), (5.3), and (5.4) in (5.1) we get $H^{*}\left(\bar{p}, F, d_{C C}, d_{C C}\right) \leq$ $(1+\bar{\epsilon})^{2}$. By the arbitrariness of $\bar{\epsilon}$ we deduce $H^{*}\left(\bar{p}, F, d_{C C}, d_{C C}\right)=1$. Finally, from Lemma 2.2 we conclude.

Acknowledgements Open access funding provided by University of Jyväskylä (JYU). The recasting of Fefferman's result from the point of view of Mostow rigidity and metric hyperbolicity was the main motivation behind this work, and was outlined by Michael Cowling, back in 2007. The authors are very grateful to both Michael Cowling and to Loredana Lanzani for several key observations that have led to a better understanding of the problem. We also want to acknowledge the thoughtfulness and care of the anonymous referees, whose suggestions we have incorporated in the paper, improving the exposition. In particular, thanks to a referee's remarks we have streamlined Sect. 3, and simplified the proof of Proposition 1.4.

Open Access This article is licensed under a Creative Commons Attribution 4.0 International License, which permits use, sharing, adaptation, distribution and reproduction in any medium or format, as long as you give appropriate credit to the original author(s) and the source, provide a link to the Creative Commons licence, and indicate if changes were made. The images or other third party material in this article are included in the article's Creative Commons licence, unless indicated otherwise in a credit line to the material. If material is not included in the article's Creative Commons licence and your intended use is not permitted by statutory regulation or exceeds the permitted use, you will need to obtain permission directly from the copyright holder. To view a copy of this licence, visit http://creativecommons.org/licenses/by/4.0/.

\section{References}

1. David, E.: Barrett, Boundary analyticity of proper holomorphic maps of domains with nonanalytic boundaries. Math. Ann. 263(4), 479-482 (1983)

2. Balogh, Zoltan M, Bonk, Mario: Pseudoconvexity and Gromov hyperbolicity. C. R. Acad. Sci. Paris Sér. I Math. 328(7), 597-602 (1999)

3. Balogh, Zoltán M, Bonk, Mario: Gromov hyperbolicity and the Kobayashi metric on strictly pseudoconvex domains. Comment. Math. Helv. 75(3), 504-533 (2000)

4. Bell, Steven, Catlin, David: Boundary regularity of proper holomorphic mappings. Duke Math. J. 49(2), 385-396 (1982)

5. Bell, Steven R: Biholomorphic mappings and the $\bar{\partial}$-problem. Ann. Math. (2) 114(1), 103-113 (1981)

6. Martin, R.: Bridson and André Haefliger, Metric Spaces of Non-positive Curvature, Grundlehren der Mathematischen Wissenschaften [Fundamental Principles of Mathematical Sciences], vol. 319. Springer, Berlin (1999)

7. Bonk, M., Heinonen, J., Koskela, P.: Uniformizing Gromov Hyperbolic Spaces, Astérisque, no. 270, viii+99 (2001)

8. Bell, Steve, Ligocka, Ewa: A simplification and extension of Fefferman's theorem on biholomorphic mappings. Invent. Math. 57(3), 283-289 (1980) 
9. Bourdon, Marc: Structure conforme au bord et flot géodésique d'un CAT(-1)-espace. Enseign. Math. (2) 41(1-2), 63-102 (1995)

10. Bonk, Mario, Schramm, Oded: Embeddings of Gromov hyperbolic spaces. Geom. Funct. Anal. 10(2), 266-306 (2000)

11. David, W.: Catlin, Estimates of invariant metrics on pseudoconvex domains of dimension two. Math. Z. 200(3), 429-466 (1989)

12. Capogna, Luca, Citti, Giovanna, Le Donne, Enrico, Ottazzi, Alessandro: Conformality and $Q$ harmonicity in sub-Riemannian manifolds. J. Math. Pures Appl. 9(122), 67-124 (2019)

13. Diederich, Klas, Fornæss, John Erik: Boundary regularity of proper holomorphic mappings. Invent. Math. 67(3), 363-384 (1982)

14. Diederich, Klas: Das Randverhalten der Bergmanschen Kernfunktion und Metrik in streng pseudokonvexen Gebieten. Math. Ann. 187, 9-36 (1970)

15. Fefferman, Charles: The Bergman kernel and biholomorphic mappings of pseudoconvex domains. Invent. Math. 26, 1-65 (1974)

16. Siqi, Fu: Asymptotic expansions of invariant metrics of strictly pseudoconvex domains. Can. Math. Bull. 38(2), 196-206 (1995)

17. Ghys, É., de la Harpe, P.: Le bord d'un espace hyperbolique, Sur les groupes hyperboliques d'après Mikhael Gromov (Bern, : Progr. Math., vol. 83. Birkhäuser Boston, Boston, MA 1990, 117-134 (1988)

18. Gromov, M.: Carnot-Carathéodory spaces seen from within, Sub-Riemannian geometry, Progr. Math., vol. 144, Birkhäuser, Basel, pp. 79-323 (1996)

19. Krantz, S.G.: Geometric Analysis of the Bergman Kernel and Metric, Graduate Texts in Mathematics, vol. 268. Springer, New York (2013)

20. Krantz, S.G.: Smoothness to the boundary of biholomorphic mappings: an overview, Function spaces in analysis, Contemp. Math., vol. 645, Amer. Math. Soc., Providence, RI, pp. 179-190 (2015)

21. Ma, Daowei: Boundary behavior of invariant metrics and volume forms on strongly pseudoconvex domains. Duke Math. J. 63(3), 673-697 (1991)

22. Nirenberg, L., Webster, S., Yang, P.: Local boundary regularity of holomorphic mappings. Comm. Pure Appl. Math. 33(3), 305-338 (1980)

23. Williams, Marshall: Geometric and analytic quasiconformality in metric measure spaces. Proc. Am. Math. Soc. 140(4), 1251-1266 (2012)

24. Andrew, M.: Zimmer, Gromov hyperbolicity and the Kobayashi metric on convex domains of finite type. Math. Ann. 365(3-4), 1425-1498 (2016)

Publisher's Note Springer Nature remains neutral with regard to jurisdictional claims in published maps and institutional affiliations. 